\title{
Manejo de residuos peligrosos en la región Cuitzeo, Michoacán, a partir de la aplicación del Método de Valoración Contingente*
}

\section{Hazardous Waste Management in the Cuitzeo Region, Michoacán, from the Application of the Contingent Valuation Method}

\author{
María Liliana Ávalos Rodríguez**, Jorge Víctor Alcaraz Vera*** \\ y José Juan Alvarado Flores****
}

\begin{abstract}
RESUMEN
La valoración económica del manejo de residuos peligrosos en México es escasa, pese a que a nivel nacional se estima una generación de 2’223,865 toneladas de residuos peligrosos de los cuales, 4,861.90 toneladas son generadas en la Región Cuitzeo, por ello, la investigación tiene como objetivo aplicar el Método y Valoración Contingente para conocer la Disposición a Pagar por el manejo ambientalmente adecuado de dichos residuos, bajo los parámetros que determina la legislación vigente. Los resultados muestran que, los generadores están dispuestos a pagar por gestiones administrativas y por cada litro, kilo o pieza de residuos peligroso que generen. Se observó, que solo un 7 por ciento de los encuestados no están dispuestos a pagar o a realizar acciones para mejorar el manejo de sus residuos. Los resultados permiten determinar que existe un desconocimiento de las obligaciones jurídicas, los tipos y volúmenes de residuos y los impactos sociales y ambientales.
\end{abstract}

Palabras clave: Manejo de residuos, valoración contingente, disposición a pagar.

Clasificación JEL: R0, Q01, Q50, Q51, Q52, Q53

\begin{abstract}
The economic valuation of the management of hazardous waste in Mexico is scarce, despite nationally is estimated a generation of 2'223,865 tons of hazardous waste, 4,861.90 tons generated in the Cuitzeo Region, therefore, the research aims to apply The Contingent Valuation Method to know the willingness to pay for the environmentally sound management of current ones, under the parameters determined by the current legislation. The results reveal that generators are willing to pay for administrative procedures and for each liter, kilo or piece of hazardous waste they generate. It is recorded that only 7 per cent of the participants are not willing to pay or take actions to improve the management of their waste. The results allow to determine that there is a lack of knowledge of legal obligations, the types and volumes of waste and the social and environmental impacts.
\end{abstract}

Keywords: Waste management, contingent valuation, willingness to pay.

JEL Classification: R0, Q01, Q50, Q51, Q52, Q53

* Fecha de recepción: 19-06-2017. Fecha de aprobación: 22-01-2018

** Universidad Michoacana de San Nicolás de Hidalgo: Correo: lic.ambientalista@gmail.com. ORCID: 0000-0002-8580-5873

*** Universidad Michoacana de San Nicolás de Hidalgo. Correo: talcarazv@hotmail.com. ORCID: 0000-0002-9115-5694

**** Universidad Michoacana de San Nicolás de Hidalgo. Correo: doctor.ambientalista@ gmail.com. ORCID: 0000-0002-5756-0960 
152 ECONOMÍA TeOría y PRÁCTICA • Nueva Época, número 48, enero-junio 2018

\section{INTRODUCCIÓN}

La generación y manejo de residuos peligrosos (RP) es un efecto de toda actividad antropogénica que busca optimizar procesos productivos y de consumo, y que su manejo inadecuado frena toda posibilidad de desarrollo al causar efectos adversos al ambiente y sociedad (Diaz, 2004), provocando distorsiones en el uso de los elementos naturales (Delacámara, 2008). Esas distorsiones son llamadas externalidades y constituyen una carga económica que debe ser atendida bajo esquemas metodológicos de prevención (OMs, 2002), sin embargo, en México se carecen de dichos esquemas para determinar el potencial de afectación y evaluar los riesgos a la salud y al ambiente (Cortinas, 2005). Los escasos estudios realizados, en particular en Michoacán, se centran en el análisis de accidentes químicos considerados como contingencias ambientales, existiendo a la fecha datos sólo del año 2000, de 72 derrames, 14 situaciones de explosión y 14 contingencias por fuego (Cofepris, 2001); además, son pocos los estudios que abordan un análisis profundo de riesgo y daño, se han encontrado escasos análisis de intoxicación por plaguicidas, arsénico, radón y plomo (Buenrostro e Israde, 2003).

Se han empleado Métodos de Valoración Económica (MVE) que bajo la premisa de resaltar el valor y no el precio del ambiente, buscan conocer la problemática y atenderla desde el enfoque de la Economía Ambiental que estudia las externalidades y la asignación intergeneracional de la responsabilidad en el cuidado del ambiente (Aguilera y Alcántara, 1994). Para el caso de las externalidades se hace uso de los métodos indirectos de valoración y se abordan problemáticas como la Disposición a Pagar (DAP) o la Disposición a Aceptar una compensación (DAA), ello para evitar un daño, disfrutar una mejora o soportar dicho daño (Almansa y Calatrava, 2001; Delacámara, 2008). Existen diversos métodos que se centran en las preferencias reveladas, sin embargo, para el presente artículo interesa el Método de Valoración Contingente (MVC) propuesto por primera vez por Ciriacy-Wantrup en 1947 como un medio para estimar la preferencia de la sociedad ante un problema ambiental. Sugirió la aplicación de este método por medio de entrevistas personales, donde se les pregunta a los individuos por su DAP o DAA (Hannemann, 1994). Dicho método ha sido aplicado en múltiples ocasiones, sin embargo, en materia de RP ha sido limitado su uso, primeramente porque los RP son poco analizados por medio de MVE, generalmente las investigaciones se han centrado en el análisis de la problemática de los Residuos Sólidos Urbanos (RSU), además, se ha observado que se analiza la DAP o DAA por parte de la sociedad quien percibe el daño o impactos de los residuos y no así por parte de quienes deben garantizar un manejo ambientalmente adecuado de los residuos, de ahí la relevancia de aplicar el MVC en materia de RP a los generadores de éstos. Respecto al análisis de los MVE 
se pueden consultar los estudios de Agüero et al. (2005); Ajata (2008); Alberini (2007); Alcalá et al. (2012); Almansa y Calatrava (2001); Buenrostro e Israde (2003); Buenrostro et al. (2007); Canchari y Ortiz (2007); Castillo et al. (2013); Cerda et al. (2010); Coutoa et al. (2013); Cho y Heo (2015); Damigos y Kaliampakos (2016); Deatona y P. Hoehn (2004); De la Parra et al. (2010); Escobar (2007); Ferreira y Marques (2015); Fierro et al. (2010); Fuentes y Serrano (2006); Gaglias et al. (2016); Gándara (2007); Geipel y Sauad (2014); Ibarrarán et al. (2003); Lladó y García (2004); Marzouk y Shumaa (2014); Ochoa (2010); Osorio y Correa (2009); P. Anex (1995); Saidón (2012) y Tzipi et al. (2005).

Existe evidencia de la viabilidad del MVC en materia de RP, a partir de la asignación de las responsabilidades intergeneracionales que aboga la Economía Ambiental. El MVC puede ser empleado para conocer la DAP por parte de quien posee en primera instancia la responsabilidad de manejar los RP correctamente, es decir, quienes los generan y no la sociedad que resiente las afectaciones. Esta premisa es la guía del presente artículo que como objetivo general busca conocer el manejo de RP en una región determinada de Michoacán a partir de la aplicación del MVC en quienes generan estos RP.

Ciertamente existen sesgos en el MVC como el hipotético que está basado en la mera suposición si fundamento, el sesgo en la información referente a si el encuestado conoce o no lo que se pretende valorar y el sesgo del entrevistador consistente cuando se exagera la DAP para quedar bien ante el encuestado (Cronin 1982, Cummings, et al. 1986). Sin embargo, dichos sesgos se reducen bajo el hecho de que se entrevistó a personas que poseen un factor común de conocimiento en el manejo de RP por contar con Número de Registro Ambiental (NRA), es decir, al cumplir con una obligación que marca la legislación mexicana en materia de RP existe la presunción de que conocen la responsabilidad legal de ser generador de RP. Con este hecho, quedan reducidos los sesgos, además de que se ha optado por preguntas abiertas en materia de DAP para evitar el sesgo de crear valoraciones auto-generados dependiendo de cómo se presenta la información (Ibarrarán et al. 2003).

Bajo este contexto, el artículo evidencia la factibilidad de emplear el MVC en materia de RP respecto a los generadores y no desde la perspectiva social, ofreciendo un panorama de qué RP se generan, cómo se manejan y la DAP por alcanzar un manejo ambientalmente adecuado de los RP. Ello porque las estimaciones oficiales muestran cifras distintas de generación de RP a las estimaciones que se han obtenido mediante la aplicación del MvC.

La Secretaría de Medio Ambiente y Recursos Naturales (Semarnat), ha estimado una generación en México - del periodo 2004 a junio del 2015- de 2'223,866 toneladas de RP, por parte de 97,348 generadores; para el caso de Michoacán, durante el mismo periodo se ha estimado una generación de 11,673.30 toneladas de 
RP, provenientes de 4,852 generadores que cuentan con NRA, de los cuales el 2 por ciento (91 registros) son grandes generadores, el 32 por ciento $(1,559)$ son pequeños y el 66 por ciento $(3,202)$ son micros. Este registro es superior al reportado por los estados de Baja California Sur, Sinaloa, Durango, Colima, Nayarit, Zacatecas, Morelos, Tlaxcala, Chiapas, Guerrero, Oaxaca, Quintana Roo y Yucatán. Respecto a la región Cuitzeo que es una de las 10 regiones de Michoacán, se ha estimado que genera el 47 por ciento de los RP reportados en el estado, es decir, 4,861.90 toneladas, el resto de las regiones han reportado 6,811.39 toneladas de RP; las actividades económicas generadores de RP son etiquetadas como: de prestación de servicios de salud (con 1,773 generadores), de servicios mercantiles (269), alimentos (43), construcción (25), metalúrgica (16), artículos de plástico (14), química (14), automotriz (1), equipos electrónicos (2), servicio de manejo de RP (8), generación de energía (7), petróleo y petroquímica (5), textiles (5), artículos metálicos (4), cemento y cal (5), congelación y productos de hielo (4), madera y sus productos (3), celulosa y papel (2), artículos y productos de diferentes materiales (2), explotación de bancos pétreos (1), comunicaciones (1), pinturas y tintas (1) y de prendas de vestir (1). Lo anterior permite suponer que la generación de RP tiene como fuentes al sector salud (clínicas, hospitales, centros médicos, veterinarias, etcétera), sin embargo, los RP con mayor rango de generación, son los enlistados como aceites usados y sólidos contaminados (Semarnat, 2016).

La región Cuitzeo es la más poblada del estado de Michoacán, alberga la quinta parte de la población de todo el estado, concentra el mayor número de generadores de RP, se ubica en ella la capital del estado y es la región que tiene mayores índices de industriales y la concentración de los servicios educativos y profesionales, la administración pública y el comercio. Está conformada por trece municipios: Acuitzio del Canje, Álvaro Obregón, Charo, Chucándiro, Copándaro, Cuitzeo, Huandacareo, Indáparapeo, Morelia, Queréndaro, Santa Ana Maya, Tarímbaro y Zinapécuaro.

\section{MATERIALES Y MÉTODOS}

Se ha empleado el MvC que tiene como finalidad conocer a través de una encuesta personal la forma en que se manejan los RP en la Región Cuitzeo de Michoacán y la DAP por un manejo ambientalmente adecuado de los RP. Se aplicó una encuesta a una muestra obtenida mediante estratificación del universo de generadores de RP en la Región que está conformado por 2,273 generadores que cuentan con NRA. La estratificación obedece a que los generadores de RP cuentan con NRA, pero de acuerdo con la legislación, existe una categorización para los generadores en micros, pequeños y grandes y con base en dicha categorización son sus obli- 
gaciones jurídicas tanto operativas como administrativas, es decir, los micro y pequeños generadores deben contar con NRA, categorización, bitácora de registro de sus RP, plan de manejo de RP, manifiestos de entrega-recepción de los RP generados, contrato con prestadores de servicio en el manejo de RP y almacén temporal para albergar sus RP; mientras que los grandes generadores, además, deberán contar con Cedula de Operación Anual (COA) y seguro ambiental (ver tabla 1). Hecha la estratificación se obtuvo una población muestral de 329 encuestas que fueron aplicadas durante el 2015.

Tabla 1. Principales obligaciones para generadores de residuos peligrosos, 2015

\begin{tabular}{|l|c|c|c|}
\hline Obligaciones de generadores & Gran & Pequeño & Micro \\
\hline Número de Registro Ambiental (NRA) & $*$ & $*$ & $*$ \\
\hline Categorización & $*$ & $*$ & $*$ \\
\hline Cédula de Operación Anual (COA) & $*$ & $*$ & $*$ \\
\hline Bitácora de registro de RP & $*$ & $*$ & $*$ \\
\hline Plan de Manejo de RP & $*$ & $*$ & $*$ \\
\hline Seguro Ambiental & $*$ & $*$ & $*$ \\
\hline Manifiestos de entrega-recepción de RP generados & $*$ & $*$ & $*$ \\
\hline $\begin{array}{l}\text { Contrato con prestadores del servicio de manejo de RP debidamente autorizados } \\
\text { por la semarnat y la sct }\end{array}$ & $*$ & $*$ & $*$ \\
\hline Almacén temporal de RP ${ }^{1}$ & $*$ & $*$ \\
\hline
\end{tabular}

${ }^{1}$ El almacén debe contar con las condiciones que señala el Reglamento de la lgpgir, como estar techado, separado de áreas comunes, restringido, con letreros alusivos a la peligrosidad de los rp resguardados, contar con ventilación e iluminación, techo, piso antiderrapante, extintor, los rp deben estar envasados de forma separada, con su letrero y características cretib, contar con fosas de retención, muros de contención, canaletas; etcétera.

Fuente: Elaboración propia con base en la lgpgir y a su reglamento, (2016).

Se elaboró una encuesta que consideró cinco grupos de variables: el grupo I referente a las variables de condición que abordan las características socioeconómicas del generador encuestado; el grupo II hace hincapié en las variables de conocimiento que aluden a las características de generación de RP y al conocimiento de los impactos ambientales y sociales; el grupo III indica las variables de cumplimiento que hacen referencia a las características técnicas del manejo de RP; el grupo IV considera las variables de cumplimiento que engloban las características operativas del manejo de RP; el grupo V se subdividió en tres apartados, el apartado $\mathrm{V}_{1}$ que aborda las variables de comprensión sobre el conocimiento de la legislación en materia de RP; el apartado $\mathrm{V}_{2}$ referente a las variables de DAP por las gestiones en materia de RP y el apartado $\mathrm{V}_{3}$ referente a las variables que abordan la DAP por la disposición por kilo/litro/pieza o equivalente de RP. 
Los datos se analizaron con apoyo del programa estadístico informático Statistical Package for the Social Sciences (SPss, por sus siglas en inglés), con la finalidad de analizar la información a partir de la estadística descriptiva y la inferencial, esta última, mediante pruebas paramétrica y no paramétrica de acuerdo con pruebas previas de normalidad que indican la distribución normal de los datos. Las pruebas de correlación empleadas fueron de Pearson y de Spearman y se realizó una regresión logística multinominal para el caso de las variables dicotómicas empleando el modelo LOGIT en relación con la DAP.

\section{RESULTADOS Y DISCUSIÓN}

\section{I.1. Análisis descriptivo de la aplicación del Método de Valoración Contingente}

Los estudios descriptivos buscan especificar las propiedades importantes de personas, grupos, comunidades o cualquier otro fenómeno que sea sometido a análisis (Dankhe, 1986). Para efectos del presente artículo, la descripción es evidente porque el instrumento de recopilación de datos, va más allá de conocer la DAP por el manejo de RP. La encuesta ofreció datos reales de qué RP se están generando y cómo se manejan.

El apartado I de la encuesta evidencia que los encuestados en porcentajes mayores al 50 por ciento son del género femenino (para los micros el 44.8 por ciento fueron del género masculino y el 55.2 por ciento del género femenino; para los pequeños el 18.7 por ciento fueron del género masculino y el 81.3 por ciento del femenino; para los grandes el 16.7 por ciento fueron del género masculino y el 83.3 por ciento del femenino). La edad promedio de los encuestados osciló entre los 18 y 72 años, observándose que todos saben leer y escribir y, los principales giros económicos son del sector salud y de mantenimiento automotriz. Además de ello, la ocupación predominante en las tres categorías del generador, es encargado (los micro muestran un 46.6 por ciento, los pequeños un 73.6 por ciento y los grandes un 50 por ciento). Por otro lado, el nivel de educación predominante, también para las tres categorías, es el nivel superior, con un 64.2 por ciento para los micros, un 35.2 por ciento para los pequeños y un 100 por ciento para los grandes. Ambas respuestas pueden suponer que quienes están encargados del manejo de RP tienen los conocimientos básicos para manejarlos adecuadamente, evitando daños al ambiente y sociedad. Respecto al ingreso de la empresa y lo que se destina al manejo de RP de dicho ingreso, es relevante el resultado obtenido, se muestra una constante en las tres categorías de generadores, porque dicen percibir menos de 50,000 
pesos mensuales; además las tres categorías en su mayoría destinan al manejo de RP menos de 1,000 mes al mes. Ello pudiera suponer que las condiciones de seguridad que actualmente se viven en la región Cuitzeo, influyeron en las respuestas de los generadores, pero a pesar de ello, las respuestas son convenientes porque brindan un esquema general de lo que perciben que destinan al manejo de RP.

El grupo II hace referencia a las variables que buscan evidenciar el conocimiento de los tipos y volúmenes de RP generados, así como los impactos al ambiente y sociedad. Los resultados muestran que los grandes generadores saben en un 100 por ciento qué es un residuo, qué es un RP, la diferencia entre residuo y RP, el volumen de generación de sus RP, las características de peligrosidad, conocidas como Corrosivas, Reactivas, Explosivas, Tóxicas, Inflamables y Biológicas (CRETIB), saben que pueden contaminar el agua, dañar la flora y fauna aledaña a la generación de RP, pueden causar daños a la salud si no se manejan adecuadamente. Es interesante apreciar que en un 83.3 por ciento saben que pueden contaminar el suelo. Respecto a los pequeños generadores se aprecia que los porcentajes se presentan por encima del 50 por ciento ( 98.9 por ciento saben qué es un residuo; el 95.6 por ciento saben qué es un RP; el 61.5 por ciento sabe la diferencia entre residuo y RP; el 95.6 por ciento sabe que genera RP; el 96.7 por ciento sabe el volumen de RP generados; el 84.6 por ciento saben las características CRETIB; el 54.9 por ciento saben que sus RP contaminan el suelo; el 69.2 por ciento sabe que pueden contaminar el agua; el 52.7 por ciento saben que puede dañar la flora y fauna y el 64.8 por ciento saben que sus RP pueden dañar la salud). Por último, los micro generadores presentan varios porcentajes por debajo del 50 por ciento, sí saben qué es un residuo (98.7 por ciento), sí saben que es un RP (en un 70.7 por ciento), sin embargo, solo un 16.4 por ciento saben las diferencias entre residuo y RP; el 52.2 por ciento saben que generan RP, sin embargo sólo el 35.8 por ciento sabe los volúmenes de RP generados; el 87.5 por ciento sabe las características CRETIB de sus RP; el 26.7 por ciento sabe que sus RP pueden contaminar el suelo; el 74.1 por ciento saben que pueden contaminar el agua; el 44 por ciento saben que pueden dañar la flora y fauna y, el 75 por ciento saben que sus RP pueden dañar la salud.

Se aprecia que los generadores identifican que generan RP sólidos como los filtros, estopas, textiles, cartón, plásticos, envases, etcétera, en proporciones distintas a las estimadas por la Semarnat. Claro está que, la estimación de la dependencia puede obedecer a los informes de los generadores quienes tienden a manifestar sólo el aceite usado como principal RP generado, sin embargo, la aplicación de este instrumento de forma personal y directa brinda información complementaria al señalar que los sólidos contaminados son los RP con que presentan una mayor generación en comparación con las estimaciones oficiales. Otro dato relevante es 
que cerca del 17 por ciento de los generadores manifiestan que sus RP los dispone como Residuos Sólidos Urbanos (RSU).

Los apartados III y IV de la encuesta fueron diseñados para conocer el grado de instrucción por parte de los generadores respecto a sus obligaciones técnicas y operativas que dispone la Ley General para la Prevención y Gestión Integral de Residuos (LGPGIR, 2003). Se puede apreciar que las tres categorías de generador saben que tienen NRA, los grandes conocen en un 100 por ciento su categorización como generadores de RP; respecto al plan de manejo los grandes muestran en su totalidad conocer que lo tienen, el 1.3 por ciento de los micros y el 47.3 por ciento de los pequeños saben que tienen dicho plan de manejo de RP. Respecto a la bitácora de registro de los RP y los manifiestos, en su mayoría los pequeños y los grandes muestran saber que sí cuentan con ellos; referente a saber si los prestadores de servicio del manejo de los RP que contratan cuentan o no con las autorizaciones debidas, sólo los pequeños generadores muestran en su mayoría saber. Además, respecto a la obligación de los grandes generadores de contar con seguro ambiental y COA, solo el 50 por ciento y el 83.3 por ciento, respectivamente saben que la tienen. Sobre las características del almacén, las respuestas demuestran que los pequeños manifiestan en su mayoría, que su almacén está techado, tiene extintor, sus RP están etiquetados, separados, con letreros alusivos a la peligrosidad, cuenta con canaletas, muros de contención, fosas de retención, pero se ubica cerca de áreas comunes; además dispone sus RP por medio de prestadores autorizados y en un 40.7 por ciento saben la siguiente fase de manejo de sus RP, dicho porcentaje es el más alto en las tres categorías de generadores. Por el contrario, los micros y los grandes generadores han afirmado en un menor porcentaje el cumplir con las condicionantes del almacén, excepto los grandes generadores que muestran un 66.7 por ciento en el cumplimiento de la separación de sus RP.

Conviene indicar que el incumplimiento de las condicionantes del almacenamiento de RP (área techada, restringida, con letreros alusivos a la peligrosidad, con extintor, con fosas de retención, muros de contención, canaletas, RP perfectamente separados, identificados y etiquetados con el nombre del residuo y sus características CRETIB, así como almacenar por menos de seis meses dichos residuos, disponerlos por conducto de prestadores de servicio debidamente autorizados, conocer la siguiente fase de manejo de sus RP y no estar cerca de áreas comunes) que prevé la LGPGIR y su Reglamento (2006) en el artículo 82, motivan la instauración de procedimientos administrativos en donde se pueden ver sancionados económicamente por cada irregularidad con multas que van desde los 30 a los 50 mil Unidades de Medida de Actualización, ello de acuerdo con la Ley Federal de Procedimiento Administrativo y a la Ley General del Equilibrio Ecológico y Protección al Ambiente. 
Respecto al almacenamiento de los RPBI, la NOM-087-Semarnat-SSA1-2002 prevé que de acuerdo con el número de camas o muestras que se realicen en el centro de salud, será su tiempo de almacenaje, determinando que en todo caso no excederá de 30 días, sin embargo, de acuerdo con los resultados se aprecia que los generadores del sector salud obligados a respetar esta norma, incumplen lo que los puede hacer acreedores a multas y clausuras.

Para los casos de que no cuentan con almacén temporal de RP, 54 por ciento de los encuestados manifestaron abiertamente que los almacenan en contenedores de basura y los disponen de la misma manera; el 14 por ciento en contenedores de plástico; el 13 por ciento en cajas de cartón; el 6 por ciento en contenedores de metal; el 4 por ciento a cielo abierto; el 3 por ciento los arrojan al drenaje y el 1 por ciento los almacenan en botes.

Las respuestas anteriores permiten asentar las bases del mercado hipotético que busca ofertar y demandar un bien, en este caso, el correcto manejo de RP, cuyo valor depende del cumplimiento de la legislación para no verse sancionados; ello evita el sesgo llamado estratégico, porque no se dan montos determinados como respuestas, se deja al albedrío del encuestado, su manifestación de cumplimiento de la normatividad que debe ser de su conocimiento porque todos ellos cuentan con un NRA previo.

El apartado V de la encuesta tuvo tres finalidades primordiales, la primera conocer qué tanto los generadores sabían de la existencia del marco jurídico en materia de RP, sí habían sido inspeccionados por la Procuraduría Federal de Protección al Ambiente (Profepa) y sí habían pagado por el acondicionamiento del almacén, asesoría jurídica y gestiones ambientales; además como segundo término, la finalidad era conocer la DAP por gestiones, acondicionamiento del almacén, asesoría jurídica y disposición de los RP, y por último, conocer los Montos que han Pagado (MHP) por todas las premisas anteriores.

Primeramente, se muestra que los grandes y pequeños generadores saben de la LGPGIR, de sus obligaciones técnicas y operativas, han sido sancionados por la Profepa y han pagado por atención jurídica y gestiones administrativas; mientras que los micros dicen que, si saben de la LGPGIR, pero sólo en un 27.6 por ciento conocen sus obligaciones técnicas y operativas, además que un porcentaje $<$ al 4 por ciento ha sido sancionado por la Profepa y han pagado por gestiones y asesoría jurídica. Lo relevante de este apartado es que las tres categorías de generadores están DAP por la gestión de trámites administrativos pendientes. Lo que muestra que a pesar de que en un 80 por ciento no cumplen las disposiciones jurídicas, están conscientes de ello y desearían estar dentro de lo que dispone la ley.

Respecto a la DAP y a los MHP por las gestiones administrativas, acondicionamiento del almacén de RP, asesoría jurídica y disposición de RP, en promedio 
los micro generadores están DAP 2,531.65 pesos, los pequeños 6,851.93 pesos y los grandes $16,350.66$ pesos. Por el contrario, los MHA por generador, se observa que los grandes y pequeños generadores han pagado más por multas ante la Profepa, asesoría y planes de manejo; mientras que los micros han pagado más por el acondicionamiento del almacén temporal de RP. Ello obedece a que los micros han mostrado desconocimiento de las obligaciones técnicas.

Se observa que los grandes generadores están DAP más de 3.00 pesos por las baterías usadas, más de 2.50 pesos por los filtros, estopas y anticongelante; más de 1.50 pesos por envases de plástico, grasas contaminadas, textiles, plásticos, lodos y cartón contaminado; los pequeños están DAP más por los filtros usados (más de 2.00 pesos por kg.); hasta 2.00 pesos por las estopas, 1.50 pesos por los cartones contaminados y menos de 1.00 peso por textiles, plástico, lodos, baterías, balatas, envases de metal, grasas y tierra contaminada. Los micros están DAP más por kilo o litro de RPBI de la categoría no anatómicos y punzocortantes, además manifiestan su DAP más por la disposición de aceite usado, cerca de 1.00 peso por litro.

La última pregunta tuvo como finalidad conocer lo que están dispuestos a realizar para manejar adecuadamente sus RP, observando que el 41 por ciento están dispuestos a acondicionar el almacén de RP con las disposiciones que prevé el artículo 82 del Reglamento de la LGPGIR; un 6 por ciento están DAP por asesoría jurídica y administrativa; un 0.20 por ciento pretenden ajustarse a programas de gobierno en materia de residuos; un 1 por ciento se asesorarán, un 10 por ciento requieren capacitación; un 0.20 por ciento analizarán la legislación; el 8 por ciento elaborarán o aplicarán un plan de manejo de RP; el 2 por ciento solicitarán incentivos fiscales por cumplir con el manejo de sus RP, el 6 por ciento solicitan cursos; el 0.20 por ciento buscarán obtener la certificación como industria limpia ante Profepa; el 0.20 por ciento realizará un análisis químico para determinar la peligrosidad de los RP; el 17 por ciento contratará la prestación de servicios de recolección y manejo de RP; el 1 por ciento requieren un diagnóstico de evaluación y cumplimiento ambiental y el 7 por ciento no hará nada para mejorar o cambiar sus condiciones de manejo de acuerdo con lo previsto en la Ley.

\section{I.2. Análisis estadístico inferencial}

Con la finalidad de saber la distribución de los datos se optó por realizar la prueba de normalidad (distribución gaussiana) que, de acuerdo con González y Lévy (2006) permite conocer la distribución de los datos, en donde para tener una distribución normal deben acercarse a 0 , sin embargo, por tratarse de datos representativos o muestrales, el rango que se considera es de -1 a 1 , dicho rango permite 
suponer la normalidad de la población. Bajo este argumento, los resultados muestran normalidad sólo entre ciertos grupos dependiendo de la categoría de los generadores, es decir, los valores de los grupos de variables para micro y pequeños generadores muestran rangos cercanos a 1, sin embargo, sólo para el grupo de variables I referente a las características socioeconómicas del generador, la significancia es $>$ a 0.05 (sig. 0.096 para micros y 0.200 para pequeños). La categoría de los grandes generadores por el contrario presenta normalidad en casi todos los grupos de variables, mostrado valores cercanos a 1 y significancias $>$ a 0.05 para los grupos I, IV, $\mathrm{V}_{2}$ y $\mathrm{V}_{3}$ (sig. 0.421, 0.680, 0.275 y 0.401 , respectivamente), salvo para los grupos II y III que a pesar de mostrar estadísticos cercanos a 1, las significancias son $<$ a 0.05 (0.031 y 0.031). Lo anterior permitió dirigir el análisis estadístico inferencial en un sentido paramétrico y no paramétrico, optando por realizar un análisis de correlación, de comparación y de predicción.

\section{I.3. Análisis estadístico inferencial de correlación}

Las tres categorías de generadores presentan buena correlación entre el grupo de variables III y IV, además, para el caso de los pequeños y grandes generadores es mejor la correlación en $r$ presentándose como buena correlación entre más grupos de variables, además, los grandes generadores muestran una excelente correlación entre los grupos de variables $\mathrm{I} \mathrm{y}_{3}$. Partiendo de estas premisas, se han realizado diversas pruebas paramétricas y no paramétricas que buscan comparar los distintos grupos de variables analizadas.

Se ha observado que los conjuntos de variables no son homogéneos entre las categorías de los generadores, y para poder hacer una predicción con mayor certeza se aplica la regresión logarítmica que permitirá conocer el comportamiento de las variables categóricas dicotómicas, por esa razón el modelo LOGIT permite aproximarse a esa inducción con base en análisis estadísticos no paramétricos.

\section{I.4. Análisis estadístico de predicción a través de la Regresión Logística}

El modelo de Regresión Logística (RL) permite conocer el comportamiento de una variable, para ello se considera el Chi-cuadrado y su P-Valor $<0.05$ que indicará en qué medida las variables explican el modelo propuesto; además se toma en cuenta el $R$ cuadrado de Cox Snell y el $R$ cuadrado de Negelkerke que indican que cuanto más alto es el $R$ cuadrado más explicativo es el modelo. El planteamiento de la RL aportará además un porcentaje que permitirá conocer el comportamien- 
to de la variable dependiente y si se desea conocer si los resultados se pueden generalizar a la población, se toma en cuenta la significancia de Chi-cuadrado y el valor de Wald. Por último, este modelo de RL permite conocer el LOGIT de predicción considerado en los valores de $\operatorname{Exp}(\mathrm{B})$, si es $<1$ indicará que a medida que aumenta el puntaje de la variable independiente va a disminuir la variable dependiente, $y$ si el valor es $>1$ indicará que si aumenta la variable independiente aumentará la dependiente también (De la Fuente, 2011).

El modelo de RL, se ha empleado con la finalidad de conocer acontecimientos en materia de DAP y manejo de RP dentro de la región Cuitzeo, ello debido a que existen variables categóricas que deben analizarse por este medio. Lo que se busca en el análisis de RL es aportar un porcentaje de predicción sobre la DAP en relación a las condiciones socioeconómicas del generador (I), el conocimiento de generación de RP (II), el cumplimiento de las disposiciones técnicas (III), así como las operativas (IV) y el conocimiento sobre la legislación en materia de RP y la DAP por un manejo ambientalmente adecuado $\left(\mathrm{V}_{1}\right)$.

Primeramente, se procede al análisis de los datos de la categoría de micro generadores y para ello conviene analizar la DAP tanto de las gestiones como de la disposición de RP, en función de las características socioeconómicas del generador. Se aprecia en el Bloque 0 que un 58.1 por ciento de los generadores pagarían más de 2,500 pesos por las gestiones en materia de RP; además, el puntaje de Wald indica que los resultados se pueden generalizar a la población (5.786), teniendo una significancia $<$ a 0.05, toda vez que es de .016. Sin embargo, presenta en la Prueba de Eficiencia Estadística de ROA un valor de 59.478, con una significancia de .105, lo que supone que las variables consideradas no aportan significativamente al modelo, siendo que valor $R$ cuadrado de Negelkerke explica el 31.6 por ciento de la varianza de la variable dependiente (.316). Se puede inducir que, si aumenta el valor de la variable independiente, es decir, las características socioeconómicas del generador, aumentará la DAP por las gestiones en materia de RP. Además de lo anterior, se aprecia que a medida que aumenta el puntaje del conocimiento sobre la generación de RP va a disminuir la DAP por las gestiones de RP, ello de acuerdo con el $\operatorname{Exp(B)~de~.270.~}$

Incluyendo más variables que el conocimiento de la generación de RP (II), como la del cumplimiento de las disposiciones técnicas (III) y operativas (IV), así como el conocimiento de la legislación y la DAP, se aprecia que cerca del 64 por ciento están DAP por las gestiones en materia de RP; si aumenta el conocimiento de las obligaciones operativas, de la legislación y de la generación de RP, se disminuiría la DAP por las gestiones; mientras si aumenta la observancia de las disposiciones técnicas aumentaría la DAP por las gestiones.

Respecto a la predicción de la DAP por las gestiones en función de los RP que se generan y los impactos al ambiente y sociedad, se observa que cerca del 63.5 
por ciento están DAP más de 2,500 pesos por las gestiones en materia de RP, de acuerdo con la Prueba de Eficiencia Estadística de ROA hay una mejora significativa en la predicción de la probabilidad de ocurrencia de las categorías de la variable dependiente (Chi-cuadrado de 16.636, con una significancia $<$ a 0.05 , siendo de .03. Por lo que el valor de $R$ cuadrado de Negelkerke indica que el modelo propuesto explica el 097 por ciento de la varianza de la variable dependiente (.097).

Además, si aumenta el valor de saber que un RP contamina el agua, la flora y fauna y se causa cáncer con el manejo indebido de sólidos contaminados, se aumentará la DAP por las gestiones en materia de RP. Mientras que, si aumenta el valor de saber que se generan RP, que éstos contaminan el suelo, saber si dañan a la salud causando intoxicación y transmisión de enfermedades por el manejo indebido de RPBI va a disminuir la DAP por el manejo de RP. Respecto al conocimiento de que los RP pueden contaminar el agua, se aprecia que más del 74 por ciento sí lo saben; además, el modelo propuesto explica en un 6 por ciento la varianza de la variable dependiente, ello en función al $R$ cuadrado de Negelkerke.

Referente a la predicción de la DAP por gestiones en función al género, se aprecia que, si la DAP por las gestiones en materia de RP están en manos del género masculino, se pagaría menos (ExpB de .253). A los microgeneradores se les cuestionó si estarían DAP por las gestiones de trámites administrativos o técnicos en materia de RP y ello en función al conocimiento sobre la generación de RP, a la observancia de las disposiciones técnicas y operativas, así como el conocimiento de la legislación, es de inducirse que el 82.8 por ciento sí estarían DAP por dichas gestiones, ese argumento está siendo explicado en un 08 por ciento con base en la varianza de la variable dependiente, ello en consideración al puntaje de $R$ cuadrado de Negelkerke. Es de apreciarse que al aplicarse un modelo de regresión logística multinominal donde se considera no pagar nada por la gestión del manejo de RP, pagar hasta 2,500 pesos y pagar más de 2,500 pesos, se observa que si aumentan el nivel de cumplimiento de las disposiciones técnicas se pagaría hasta 2,500 pesos por dichas gestiones. Argumento que puede extrapolarse a la población por obtener una significancia $<$ a 0.05 , siendo de .005 .

Por último, se busca conocer la DAP por la disposición ambientalmente adecuada de RP, para ello se aplica el modelo de regresión lineal binaria con las condiciones socioeconómicas del micro generador, observándose que el 76.2 por ciento están DAP hasta 5 pesos por kilo/litro/pieza de RP generados, apreciándose que, si aumenta el grado de estudios, mejora la ocupación, aumenta el ingreso mensual considerado de la empresa, mejora la vivienda y la atención médica, se aumentará la DAP por RP. Ello de acuerdo con la tabla de las variables de la ecuación de la prueba, que además puede generalizarse a la población por tener una significancia $<$ a 0.05 , además el valor de $R$ cuadrado de Negelkerke indica que 
el modelo propuesto explica el 54.7 por ciento de la varianza de la variable dependiente $(.547)$.

En otro modelo de RL se aprecia que el 63.4 por ciento está DAP por RP hasta 5 pesos y que si aumenta el conocimiento de la generación de RP, el nivel de cumplimiento de las disposiciones operativa y el conocimiento de la legislación, aumentará dicha DAP por la disposición ambientalmente adecuada de los RP; ello queda justificado y puede ampliarse a la población porque tiene un Chi-cuadrado de 15.969 con una significancia por debajo de 0.05 , siendo de 0.003 y el $R$ cuadrado de Negelkerke explica el 11.8 por ciento de la varianza de la variable dependiente (DAP por RP).

Referente a la DAP por RP en función al conocimiento de generar RP y los impactos que éstos producen en el ambiente y sociedad, mediante un modelo de RL se aprecia que el 62.8 por ciento está DAP por RP hasta 5 pesos y para aumentar la DAP tendría que aumentar el conocimiento sobre la generación de RP, saber cómo y por qué contaminan el suelo y como daña a la salud. Ello puede generalizarse a la población toda vez que el valor de Chi-cuadrado es de 19.972, con una significancia de 0.10 que resulta ser un $\mathrm{P}<0.05$; y de acuerdo con el $R$ cuadrado de Negelkerke el modelo propuesto explica el 14.6 por ciento de la varianza de la variable dependiente.

Respecto a la aplicación del Modelo de RL aplicado en datos de pequeños generadores de RP se observa que al realizar un modelo de RL en consideración con la DAP por gestiones en función del grado de estudios, ocupación, ingreso mensual considerado de la empresa, ingreso destinado al manejo de RP, vivienda y atención médica, se aprecia que en el Bloque 0 el valor previsto de la DAP en función de dichas variables es de 65.2 por ciento de que se pague hasta 6,500 pesos, sin embargo, el valor de Chi-cuadrado es de 23.236 que indica que no es significativa la relación, debido a que resulta el P-Valor $>$ a 0.05 , siendo de .079 de lo que se puede deducir que las variables independientes no explican la DAP por las gestiones en materia de RP. Al respecto el $R$ cuadrado de Negelkerke es de .317 , lo que indica que en un 31.7 por ciento el modelo explica la varianza de la variable dependiente. Respecto a la tabla de clasificación, del bloque 1, se da un valor inferido del 74.2 por ciento de funcionabilidad del modelo, resaltando que si aumenta el nivel de estudios, ocupación e ingreso de la empresa va a aumentar el valor de la DAP por las gestiones, ello con base en ell valor de $\operatorname{Exp}(\mathrm{B})$, de la misma manera dicho valor indica que la vivienda y la atención médica no aportan al modelo.

El modelo de RL también se aplicó en consideración a la DAP por las gestiones en función al conocimiento de la generación de RP y los efectos que ocasiona al ambiente y sociedad. Los resultados indican que en un 67.4 por ciento estarían 
DAP por las gestiones en materia de RP hasta 6,500 pesos de acuerdo con las variables independientes consideradas, aunque la significancia mostrada de Chi-cuadrado es $>$ a 0.05 , toda vez que indica un valor de .96 , mientras que el valor de $R$ cuadrado de Negelkerke infiere que en un 11.5 por ciento se explica la varianza de la variable dependiente. Valores que resultan inferiores y que permiten concluir que el grado de conocimiento de la generación de RP y sus impactos al ambiente y sociedad no son determinantes en la DAP por las gestiones en el manejo de RP. Sin embargo, los valores de $\operatorname{Exp}(B)$ permite inferir que, si se sabe el daño en la flora y fauna, así como en la salud, se puede aumentar la DAP por las gestiones en el manejo de RP.

Se buscó conocer la DAP por las gestiones en materia de RP en función del conocimiento del manejo de RP, el cumplimiento de las disposiciones técnicas, operativas y el conocimiento de la legislación en materia de RP, sin embargo, el modelo de RL indica que en un 65.2 por ciento se estaría DAP hasta 6,500 pesos en función de las variables independientes consideradas, pero resultan no ser significativas debido al P-Valor $>$ a 0.05 que arroja el Chi-cuadrado, que es de .192, mientras que el puntaje de R cuadrado de Negelkerke es de tan sólo .071, lo que indica que las variables independientes explican en un 0.7 por ciento la varianza de la variable dependiente. Además, con base en el valor de $\operatorname{Exp}(B)$ que permite inferir el comportamiento de las variables independientes, se aprecia que, si aumenta el conocimiento de las disposiciones técnicas en materia de RP, aumentará la DAP por las gestiones de dichos RP. Sin embargo, si aumenta el conocimiento de la generación de RP y sus impactos al ambiente y sociedad, la observancia de las obligaciones técnicas y el conocimiento de la legislación, no aumentará la DAP por las gestiones. Esto mismo puede inferirse con el modelo de RL planteado entre la variable dependiente del cumplimiento de las disposiciones técnicas (III) en función con el conocimiento de la generación de RP y sus impactos (II), así como con el conocimiento de la legislación $\left(\mathrm{V}_{1}\right)$, dando como resultado una significancia < a .05 , toda vez que tiene un valor de .001 , con un puntaje en Chi-cuadrado de 13.348. Mientras que el valor de $R$ cuadrado de Negelkerke indica que en un 21 por ciento (.210) el modelo propuesto explica a la varianza de la variable dependiente (III). Así mismo, se buscó conocer el comportamiento de la variable III con las condiciones socioeconómicas del generador (estudios, ocupación, ingreso mensual de la empresa, el ingreso de la empresa destinado al manejo de RP), dando como resultado que en un 78 por ciento se cumpliría con las disposiciones técnicas del manejo de RP, toda vez que la significancia de Chi-cuadrado con valor de 18.5543 es de .029 , lo que resulta $<$ a .05 . El valor de $R$ cuadrado de Negelkerke es de .283 , lo que indica que en un 28.3 por ciento el modelo explica la varianza de la variable dependiente (III). Y de forma predictiva se puede argumentar que 
por el valor de $\operatorname{Exp}(\mathrm{B})$, si aumenta el ingreso de la empresa y el monto destinado al manejo de RP, así como el grado de estudios y la ocupación aumentará el nivel de cumplimiento de las obligaciones técnicas en materia de RP.

Se buscó conocer la predicción de la DAP por el manejo ambientalmente adecuado de RP en función a las características socioeconómicas del generador (I), el conocimiento de generación y sus impactos (II) y el cumplimiento de las obligaciones (III, IV y $\mathrm{V}_{1}$ ) arrojando como resultados que en un 62.9 por ciento se pagaría más de 10 pesos por el DAP por el manejo de RP, ello en consideración a las variables independientes propuestas; además, la significancia es baja, pero se mantiene por encima del P-valor considerado, de .05. Respecto al puntaje de $R$ cuadrado de Negelkerke, se aprecia un valor de .278, lo que indica que en un 27.8 por ciento el modelo explica la varianza de la variable dependiente. Además, es factible inferir de acuerdo con el valor de $\operatorname{Exp}(\mathrm{B})$ que, si aumenta el grado de estudios y la ocupación, aumentará la DAP por el manejo de los RP.

Respecto al comportamiento de la variable dependiente (DAP por el manejo de $\mathrm{RP})$ en función a la variable II, se aprecia que la significancia de Chi-cuadrado es de .061, lo que indica que no es notoriamente significativa la predicción con base en las variables consideradas, presentando un puntaje de R-cuadrado de Negelker$k e$ de .216 , lo que indica que la varianza de la variable dependiente se explica en un 21.6 por ciento con base en el modelo considerado. Sin embargo, resulta efectivo el modelo de RL planteado entre la DAP por RP con las variables III, IV y $\mathrm{V}_{1}$, dando un 62.9 de disponibilidad a pagar más de 10 pesos con base en estas variables, teniendo una significancia de Chi-cuadrado $<$ a 0.05 , siendo de .038 , con un $R$ cuadrado de Negelkerke de .155, (15.5 por ciento) que explica la varianza de la variable dependiente. Además, con el valor de $\operatorname{Exp}(B)$ se puede inferir que si aumentan todas las variables independientes (III, IV y $\mathrm{V}_{1}$ ) aumentará la DAP por el manejo ambientalmente adecuado de los RP.

Por último, aplicando el modelo de RL para los grandes generadores es conveniente mencionar que los datos son peculiaridades de homogeneidad, por lo que los resultados de algunos modelos de RL se limitan a las variables que no presenten redundancias. Para el caso de la predicción de la DAP por gestiones en materia de RP en función al cumplimiento de las obligaciones técnicas (III), operativas (IV) y conocimiento de la legislación, así como DAP $\left(\mathrm{V}_{1}\right)$, se aprecia que en un 83.3 por ciento estarían DAP por las gestiones, hasta 17,000 pesos, ello de acuerdo con los datos reportados en el Bloque 1 del modelo; sin embargo, el P-Valor es $>$ a 0.05, arrojando un puntaje de .148, con un valor en Chi-cuadrado de 3.819. Mientras que el valor de $R$ cuadrado de Negelkerke es de .628, lo que indica que en un 62.8 por ciento el modelo explica la varianza de la variable dependiente. Además, el valor de $\operatorname{Exp}(B)$ dice que, si aumenta la observancia de las responsabilidades téc- 
nicas, aumentará la DAP por las gestiones técnicas. Referente al modelo que busca conocer la DAP por las gestiones en función a los estudios, ocupación e ingreso, es pertinente mencionar que en consideración a estas variables independientes, cerca del 50 por ciento estarían DAP por las gestiones más de 17,000 pesos; además, se observa que el modelo presenta en el Bloque 1 un Chi-cuadrado de 8.318, con una significancia de .081, siendo el valor de R-cuadrado de Negelkerke de 1.000, lo que indica que en un 100 por ciento el modelo está explicando la varianza de la variable dependiente.

En esta misma tesitura, se encuentra la DAP por el manejo de RP en consideración a los estudios, ocupación e ingreso, infiriendo un porcentaje global del 66.7 por ciento que estarían DAP por el manejo de los RP más de 15 pesos por kilo, litro o pieza. Además, se aprecia un valor de $R$-cuadrado de Negelkerke de 1.000, lo que indica que en un 100 por ciento las variables independientes explican a la variable dependiente. Referente a la DAP por el manejo de RP se aprecia un porcentaje de predicción del 83.3 por ciento en función de las variables II (conocimiento de la generación de RP y sus impactos), III (cumplimiento a las disposiciones técnicas), IV (cumplimiento a las disposiciones operativas) y $\mathrm{V}_{1}$ (conocimiento de la legislación y DAP). Además, el valor de R-cuadrado de Negelkerke indica que en un 56.6 por ciento (.566) las variables independientes explican a la variable dependiente. Se aprecia que el valor de $\operatorname{Exp}(B)$ indica que, si aumenta el conocimiento de la generación de RP y sus impactos, así como el del cumplimiento de las obligaciones técnicas, aumentará la DAP por el manejo ambientalmente adecuado de los RP.

\section{Conclusiones}

Mediante la aplicación de MVC se pudo conocer el manejo de los RP en la Región Cuitzeo, Michoacán, apreciando que éste se realiza en gran medida en desapego a los principios de la LGPGIR, es relevante observar cómo los generadores de RP a pesar de conocer sus obligaciones técnicas y operativas, como contar con NRA, categorización como micro, pequeño o gran generador, plan de manejo, bitácora de registro de RP del almacén, COA, seguro ambiental y contratación de prestadores de servicio de la recolección, transporte, acopio y disposición final de los RP (incineración, tratamiento, reciclaje, co-procesamiento y/o confinamiento controlado), además de contar con un área destinada al resguardo de los RP generados, manifiestan manejar de forma inadecuada los RP que generan, en primera instancia por reportar ante la Semarnat sólo algunos de los RP que generan, principalmente el aceite usado, además, argumentan manejar los RP como RSU, situación que trae como consecuencia alterar la cadena de responsabilidad compartida pero diferen- 
ciada en el manejo de RP. Se ha observado que los generadores desconocen los alcances de la LGPGIR y las consecuencias que deriva su inobservancia, es importante resaltar que los micro generadores presentan un mayor rezago en el conocimiento de los impactos al ambiente y sociedad por el manejo indebido de sus RP.

De igual manera es conveniente resaltar que a pesar de que pagan menos de 1,000 pesos por el manejo de los RP generadores, los encuestados manifiestan estar DAP por mejorar sus condiciones de manejo y disposición de RP, incluso es relevante indicar que están dispuestos a realizar actividades paralelas para alcanzar mejores condiciones de manejo de sus RP, sólo un 7 por ciento manifiesta no estar dispuesto a realizar ningún cambio en su actual proceder.

Se concluye, que el MVC fue útil para conocer el manejo de los RP en la región estudiada y dirigido a quienes generan los RP y no al sector social como comúnmente se emplea, hecho que permite argumentar que dicho método es factible de replicarse en otros estudios que analicen otros residuos como RSU y residuos de manejo especial (RME) con el enfoque del generador.

\section{REFERENCIAS BibLIOGRÁFICAS}

Agüero, A. A.; Carral, M.; Sauad, J. J. y Yazlle, L. L. (2005), “Aplicación del método de valoración contingente en la evaluación del sistema de gestión de residuos sólidos domiciliarios en la ciudad de Salta, Argentina", Revista Iberoamericana de Economía Ecológica, vol. 2, pp. 37-44.

Aguilera Klink, Federico y Alcántara, Vicent (comps.) (1994), De la economía ambiental a la economía ecológica, Barcelona, Icaria, Fuhem, 408, p. 21.

Ajata Arghata, Claudia (2008), Aplicación del método de valoración contingente por la disponibilidad a pagar en el servicio de aseo urbano en la localidad de Huanuni del departamento de Oruro, Universidad Técnica de Oruro, Facultad de Ciencias Económicas, Financieras y Administrativas, Bolivia.

Alberini, Annay y Frost, Shelby (2007), "Forcing Firms to Think About the Future: Economic Incentives and the Fate of Hazardous Waste", Environmental and Resource Economics, 36, pp. 451-474, Springer, 2006.

Alcalá Valdivia, Ramón; Abelino Torres, Gonzalo; López, Santiago Marco A. y Zavala Pineda, María J. (2012), "Valoración económica del reciclaje de desechos urbanos", Revista Chapingo, vol. 18, núm. 3, agosto-diciembre 2012, http://dx.doi.org/10.5154/r.rchscfa.2010.07.044.

Almansa C. y Calatrava, J. (2001), Aplicación del método de valoración contingente a la valoración económica de las externalidades generadas en los 
proyectos de restauración hidrológica forestal, Departamento de Economía y Sociología Agrarias de la Junta de Andalucía. Centro de Investigación y Desarrollo Agrario, Granada.

Anex, Robert P. (1995), “A Travel-Cost Method of Evaluating Household Hazardous Waste Disposal Services", Journal of Environmental Management, 45, pp. 189-198.

Buenrostro, Otoniel e Israde, Isabel (2003), "La gestión de los residuos sólidos municipales en la cuenca del lago de Cuitzeo, México", Revista Internacional de Contaminación Ambiental, 19 (4), pp. 161-169.

Buenrostro Delgado, Otoniel; Ojeda Benítez, Sara y Márquez Benavides, Liliana (2007), "Comparative analysis of hazardous household waste in two Mexican regions", Waste Management, 27, pp. 792-801.

Canchari Silverio, Godelia y Ortiz Sánchez, Oswaldo (2007), "Valorización de los residuos sólidos en la ciudad universitaria de la Universidad Nacional Mayor de San Marcos", Revista del Instituto de Investigaciones FIGMMG, vol. 11, núm. 21, pp. 95-99.

Cardona Madariaga, D. F.; González Rodríguez, J. L.; Rivera Lozano, M. y Cárdenas Vallejo, E. (2013), Inferencia estadística-Módulo de regresión lineal simple, Documento de investigación núm. 47, Universidad del Rosario, Escuela de Administración Editorial Universidad del Rosario, Bogotá.

Castillo Ponce, Ramón A.; Camargo Negrete, Gustavo y Rodríguez Espinosa, María de Lourdes (2013), "La disposición de residuos peligrosos en la frontera norte de México: El caso de Baja California", Estudios Fronterizos, nueva época, vol. 14, núm. 27, enero-junio.

Cerda, Arcadio; García, Leidy; Bahamondez, Alejandra y Poblete, Viviana (2010), "Disposición a pagar para mejorar la calidad del aire en Talca, Chile: comparación entre usuarios y no usuarios de chimeneas a leña", Red de Revistas Científicas de América Latina, el Caribe, España y Portugal, Sistema de Información Científica.

Cho, S.; Kim, J.; Park, H. C., y Heo, E. (2015), "Incentives for waste cooking oil collection in South Korea: a contingent valuation approach", Resources, Conservation and Recycling, 99, pp. 63-71.

Cortinas de Nava, Cristina (2005), Implicaciones de la regulación de los residuos peligrosos, Semarnat, p. 3. 
Coutoa, Nuno; Valter, Silva; Monteirob, E. y Rouboaa, A. (2013), "Hazardous waste management in Portugal: An overview”, Energy Procedia, 36, pp. 607-611.

Cronin, F. J. (1982), “The household's decision to accept or reject a conditional transfer offer", Southern Economic Journal, pp. 218-234.

Cummings, R. G.; Brookshire, D. S. y Schulze, W. D. (1986), Valuing Public Goods: An Assessment of the Contingent Valuation Method, Totowa, NJ, Rowman and Allenheld.

Damigos, D.; Menegaki, M. y Kaliampakos, D. (2016), "Monetizing the social benefits of landfill mining: Evidence from a Contingent Valuation survey in a rural area in Greece", Waste Management, 51, pp. 119-129.

Dankhe, G. L. (1986), "Investigación y Comunicación”, en Fernández-Collado, C. y Dankhen, G. L. (eds.), La comunicación humana, Ciencia social. México, Mc Graw-Hill, pp. 385-454.

Deatona, B. James y Hoehn, John P. (2004), "Hedonic analysis of hazardous waste sites in the presence of other urban disamenities", www.elsevier. com/locate/envsci, Environmental Science y Policy, 7, pp. 499-508.

Delacámara, Gonzalo (2008), "Guía para decisores: Análisis económico de externalidades ambientales", Comisión Económica para América Latina y el Caribe (CEPAL). Desarrollado en el contexto del Programa de Cooperación entre el Ministerio Federal de Cooperación Económica y Desarrollo de Alemania (BMZ), a través de su agencia de cooperación Deutsche Gesellschaft für Technische Zusammenarbeit (GTZ) y la CEPAL, llamado "Modernización del Estado, desarrollo productivo y uso sostenible de recursos naturales", GER/05/001.

De la Fuente Fernández, S. (2011), Regresión Logística. España, Facultad de Ciencias Económicas y Empresariales, UnAM-Universidad Autónoma de Madrid.

De la Parra Rentería, Carlos A.; Rodríguez Lepure, Ana L. y Pacheco Pedraza, Alberto (2010), "Disposición a pagar por el servicio de limpia en la ciudad de Tijuana”, Diagnóstico de los residuos sólidos urbanos en Tijuana, Baja California, El Colegio de la Frontera Norte.

Escobar, Ninel (2007), “¿Qué determina la imposición de multas?: Evidencia del Programa Federal de Inspección Industrial en la ciudad de México", Gaceta de Economía, Instituto Nacional de Ecología, año 16, núm. especial, t. I. 
Ferreira, S. y Marques, R. C. (2015), "Contingent valuation method applied to waste management”, Resources, Conservation and Recycling, 99, pp. 111-117.

Fierro Ochoa, Aurora; Armijo de Vega, Carolina; Buenrostro Delgado, Otoniel y Valdez Salas, Benjamín (2010), "Análisis de la generación de residuos sólidos en supermercados de la ciudad de Mexicali, México", Revista Internacional de Contaminación Ambiental, vol. 26, núm. 4.

Fuentes Perea, Luz Amparo y Serrano Pedraza, Ana Milena (2006), Valoración económica de los impactos socioeconómicos y ambientales ocasionados por el manejo de los residuos sólidos urbanos en el relleno sanitario "La Esmeralda” del municipio de Barrancabermeja: Aplicación del Método Multicriterio, Universidad Industrial de Santander Escuela de Economía y Administración Facultad de Humanidades, Bucaramanga.

Gaglias, A.; Mirasgedis, S.; Tourkolias, C. y Georgopoulou, E. (2016), “Implementing the Contingent Valuation Method for supporting decision making in the waste management sector", Waste Management, 53 pp. 237-244.

Gándara Fierro, Guillermo (2007), Valoración monetaria de la contaminación por incineración y vertido de residuos sólidos urbanos, Instituto Tecnológico y de Estudios Superiores de Monterrey.

Geipel, Verónica y Sauad, Juan José (2014), "Valoración económica y financiera de la Gestión Integral de Residuos Sólidos Urbanos en el Valle de Siancas. Municipio de El Bordo", Revista de Divulgación, 01, Escuela de Recursos Naturales Ingeniería en Recursos Naturales y Medio Ambiente, ECONAMIA.

González, N.; Abad, J. y Lévy, J. P. (2006), "Normalidad y otros supuestos en análisis de covarianzas", Modelización con estructuras de covarianza en Ciencias Sociales. Temas esenciales, avanzados y aportaciones especiales, pp. 31-58.

Hannemann, Michael W. (1994), "Valuing the environment through contingent valuation", Journal of Economic Perspectives, vol. 8 (4), pp. 19-43.

Ibarrarán, María Eugenia; Islas, Iván y Mayett, Eréndra (2003), "Valoración económica del impacto ambiental del manejo de residuos sólidos municipales: estudio de caso", Gaceta Ecológica, núm. 67, abril-junio, pp. 69-82, México, Semarnat, Red de Revistas Científicas de América Latina, el Caribe, España y Portugal. Sistema de Información Científica,

LGPGIR (08 de octubre de 2004), Ley General para la Prevención y Gestión Integral de Residuos. México, Camara de Diputados. 
172 ECONOMÍA TeORÍA Y PRÁCTICA • Nueva Época, número 48, enero-junio 2018

Lladó, Verdejo; Agenor, Alberto y García Rodríguez, José Félix (2004), “Costo efectividad en el manejo de los residuos peligrosos biológico infecciosos en un Hospital General”, Red de Revistas Científicas de América Latina, el Caribe, España y Portugal Sistema de Información Científica.

Marzouk, Mohamed y Shimaa, Azab (2014), "Environmental and economic impact assessment of construction anddemolition waste disposal using system dynamics", Contents lists available at ScienceDirect Resources, Conservation and Recycling journal h om epa ge: www.elsevier.com/locate/resconrec.

NOM-087-ECOL-SSA1-2002, Protección ambiental-salud ambiental residuos peligrosos biológico-infecciosos-clasificación y especificaciones de manejo.

Ochoa Guarachi, Lucia Dora (2010), Valoración económica de los factores relacionados al reciclaje en el municipio de El Alto, Universidad Mayor de San Andrés, Facultad de Ciencias Económicas y Financieras, Bolivia.

Organización Mundial de la Salud (oms) (2002), Preparemos Ambientes Saludables para los Niños.

Osorio Múnera, Juan David y Correa Restrepo, Francisco Javier (2009), “Un análisis de la aplicación empírica del método de valoración contingente", Redalyc, Sistema de Información Científica Red de Revistas Científicas de América Latina, el Caribe, España y Portugal.

Reglamento de la Ley General Para la Prevención y Gestión Integral de los Residuos (2006), Diario Oficial de la Federación, 30 de noviembre.

Saidón, Mariana (2012), "Environmental valuation of waste recycling: The case of Quilmes, Argentina”, Economía, XXXVII, 34 (julio-diciembre), pp. 33-53.

Secretaría de Medio Ambiente y Recursos Naturales (Semarnat)/Dirección General de Gestión Integral de Materiales y Actividades Riesgosas (DGGIMAR) de la Semarnat, 2016.

Tzipi Eshet, Ofira; Ayalon, Mordechai Shechter (2005), "Valuation of externalities of selected waste management alternatives: A comparative review and analysis", Resources, Conservation and Recycling, 46 (2006), pp. 335-364. 